\title{
Geometrothermodynamics of Myers-Perry Black Holes
}

\author{
Alessandro Bravetti, ${ }^{1,2}$ Davood Momeni, ${ }^{3}$ Ratbay Myrzakulov, ${ }^{3}$ and Aziza Altaibayeva ${ }^{3}$ \\ ${ }^{1}$ Dipartimento di Fisica and ICRA, "Sapienza" Università di Roma, Piazzale Aldo Moro 5, 00185 Rome, Italy \\ ${ }^{2}$ Instituto de Ciencias Nucleares, Universidad Nacional Autónoma de México, AP 70543, 04510 México, DF, Mexico \\ ${ }^{3}$ Eurasian International Center for Theoretical Physics, L.N. Gumilyov Eurasian National University, Astana 010008, Kazakhstan \\ Correspondence should be addressed to Alessandro Bravetti; bravetti@icranet.org
}

Received 13 March 2013; Revised 4 June 2013; Accepted 5 June 2013

Academic Editor: Rong-Gen Cai

Copyright (C) 2013 Alessandro Bravetti et al. This is an open access article distributed under the Creative Commons Attribution License, which permits unrestricted use, distribution, and reproduction in any medium, provided the original work is properly cited.

\begin{abstract}
We consider the thermodynamics and geometrothermodynamics of the Myers-Perry black holes in five dimensions for three different cases, depending on the values of the angular momenta. We follow Davies approach to study the thermodynamics of black holes and find a nontrivial thermodynamic structure in all cases, which is fully reproduced by the analysis performed with the techniques of Geometrothermodynamics. Moreover, we observe that in the cases when only one angular momentum is present or the two angular momenta are fixed to be equal, that is, when the thermodynamic system is two dimensional, there is a complete agreement between the divergences of the generalized susceptibilities and the singularities of the equilibrium manifold, whereas when the two angular momenta are fully independent, that is, when the thermodynamic system is three dimensional, additional singularities in the curvature appear. However, we prove that such singularities are due to the changing from a stable phase to an unstable one.
\end{abstract}

\section{Introduction}

Black holes are very special thermodynamic systems. They are thermodynamic system since they have a temperature, the celebrated Hawking temperature [1], and a definition of entropy via the Bekenstein area law [2], from which one can prove that the laws of thermodynamics apply to black holes [3]. On the other side, they are very special thermodynamic systems, and since, for instance, the entropy is not extensive, they cannot be separated into small subsystems, and perhaps the worst fact, their thermodynamics does not possess a microscopic description yet (see, e.g., [4] for a clear description of these problems).

In this puzzling situation, one of the most successful and at the same time discussed approach to the study of black holes phase transitions is the work of Davies [5]. According to Davies, black holes can be regarded as ordinary systems, showing phase transitions right at those points where the generalized susceptibilities, that is, second-order derivatives of the potential, change sign most notably through an infinite discontinuity. Since there is no statistical mechanical description of black holes as thermodynamic systems, it is hard to verify Davies approach with the usual technique of calculating the corresponding critical exponents (although very interesting works on this subject exist, see, e.g., [4, 618]). In fact, the main drawback of this approach is that one has to choose arbitrarily the order parameter for black holes.

A possible resolution to this situation can then come from the use of thermodynamic geometry. Since the pioneering works of Gibbs [19] and Carathéodory [20], techniques from geometry have been introduced into the analysis of thermodynamics. In particular, Fisher [21] and Radhakrishna Rao [22] proposed a metric structure in the space of probability distributions which has been extensively used both in statistical physics and in economics (for a recent review see [23]). Later, Weinhold [24] introduced an inner product in the equilibrium space of thermodynamics based on the stability conditions of the internal energy, taken as the thermodynamic potential. The work of Weinhold was then developed by Ruppeiner [25] from a different perspective. Ruppeiner moved from the analysis of fluctuations around equilibrium and from the gaussian approximation of the probability of fluctuations and found a thermodynamic metric which is defined as (minus) the hessian of the entropy 
of the system. Remarkably, Ruppeiner geometry was found to be conformally related to the one proposed by Weinhold. Moreover, Ruppeiner metric is intrinsically related to the underlying statistical model, and in fact the scalar curvature of the Riemannian manifold representing the system using Ruppeiner metric happens to have exactly the same exponent as the correlation volume for ordinary systems (see, e.g., [26] for a review).

All these approaches have been widely used to study ordinary systems, and in particular Ruppeiner metric has been also used to study many black holes configurations (see [27] and refernces therein). This is because one can argue that being Ruppeiner metric defined only from thermodynamic quantities and on the other side giving information about the statistical model, then it can provide some hints towards the resolution of the long standing problem of understanding the microscopic properties of black holes (see, e.g., [27]).

On the other side, the problem with the use of thermodynamic geometries to study black holes thermodynamics is that black holes are not ordinary systems, as we argued previous. For instance, Ruppeiner metric in many cases gives exactly the same results of Davies approach (which is based upon ordinary thermodynamics), while in some other important cases it does not converge to the same results, as it happens for example, in the Reissner-Nordström and Kerr cases (see, e.g., [27-29]). One can argue either that Davies approach is inaccurate or that the application of Ruppeiner metric to black holes may be imperfect, due to the strange nature of black holes as thermodynamic systems. In fact, there is still an open debate on this topic (see, e.g., the discussion in $[27,30])$.

Furthermore, in the recent years, a new approach in the context of thermodynamic geometry has been proposed by Quevedo [31], known as geometrothermodynamics (GTD). According to this approach, the Riemannian structure to be introduced in the equilibrium space should be consistent with the property of Legendre invariance, a property which is at the core of ordinary thermodynamics. In GTD some families of metrics have been found that share the Legendre invariance property, and they have also been proven to interpret in a consistent manner the thermodynamic properties of ordinary systems, chemical interactions, black holes configurations, and cosmological solutions (see [31-39]). In particular, the correspondence between the divergences of the scalar curvature of the equilibrium manifold of GTD and the phase transition points signaled by the divergences of the heat capacity (i.e., phase transitions à la Davies) seems to be a general fact, according to the variety of systems analyzed so far and to the general expressions given in [40]. In addition, a recent study [41] of the thermodynamics of the ReissnerNordström and Kerr black holes in any dimensions suggested that the GTD approach can detect not only the points of phase transitions due to singularities of the heat capacities but also divergences of the full spectrum of the generalized susceptibilities.

On the other side, the thermodynamic properties of the Myers-Perry black holes in five dimensions have been extensively studied in the literature from completely different points of view (see, e.g., [4, 29, 42-46]). In this work, we give special emphasis on the relation between divergences of the generalized susceptibilities and curvature singularities of the metric from GTD. For example, we do not consider here possible phase transitions related to change in the topology of the event horizon, an intriguing question which was addressed, for example, in [4]. We find out that the GTD thermodynamic geometry is always curved for the considered cases, showing the presence of thermodynamic interaction and that its singularities always correspond to divergences of the susceptibilities or to points where there is a change from a stable to an unstable phase. This will allow us to infer new results on the physical meaning of the equilibrium manifold of GTD.

The work is organized as follows. In Section 2, we present the basic aspects of GTD and introduce all the mathematical concepts that are needed. In Section 3, we perform the parallel between the thermodynamic quantities and the Geometrothermodynamic description of the fivedimensional Myers-Perry black holes for three different cases, depending on the values of the angular momenta. Finally, in Section 4, we comment on the results and discuss possible developments.

\section{Basics of Geometrothermodynamics}

Geometrothermodynamics (GTD) is a geometric theory of thermodynamics recently proposed by Quevedo [31]. It makes use of contact geometry in order to describe the phase space $\mathscr{T}$ of thermodynamic systems and express the first law of thermodynamics in a coordinate-free fashion. Furthermore, GTD adds a Riemannian structure $G$ to the phase space and requests $G$ to be invariant under Legendre transformations, in order to give it the same properties which one expects for ordinary thermodynamics. Moreover, GTD introduces the manifold of the equilibrium space $\mathscr{E}$ as the maximum integral submanifold of the contact structure of $\mathscr{T}$, characterized by the validity of the first law of thermodynamics [31]. At the same time, GTD prescribes also to pull back the Riemannian structure $G$ to the equilibrium space. This results in a naturally induced Riemannian structure $g$ in $\mathscr{E}$, which is supposed to be the geometric counterpart of the thermodynamic system. Such a description has been proposed in order to give thermodynamic geometry a new symmetry which was not present in previous approaches, that is, the Legendre invariance.

Let us see now the mathematical definitions of the GTD objects that we shall use in this work. If we are given a system with $n$ thermodynamic degrees of freedom, we introduce first a $(2 n+1)$-dimensional space $\mathscr{T}$ with coordinates $Z^{A}=$ $\left\{\Phi, E^{a}, I^{a}\right\}$, with $A=0, \ldots, 2 n$ and $a=1, \ldots, n$, which is known as the thermodynamic phase space [31]. We make use of the phase space $\mathscr{T}$ in order to correctly handle both the Legendre transformations and the first law of thermodynamics. In fact, in classical thermodynamics, we can change the thermodynamic potential using a Legendre transformation, which is defined in $\mathscr{T}$ as the change of coordinates given by [47]:

$$
\left\{\Phi, E^{a}, I^{a}\right\} \longrightarrow\left\{\widetilde{\Phi}, \widetilde{E}^{a}, \widetilde{I}^{a}\right\}
$$




$$
\begin{gathered}
\Phi=\widetilde{\Phi}-\delta_{k l} \widetilde{E}^{k} \widetilde{I}^{l}, \quad E^{i}=-\widetilde{I}^{i}, \quad E^{j}=\widetilde{E}^{j}, \\
I^{i}=\widetilde{E}^{i}, \quad I^{j}=\widetilde{I}^{j},
\end{gathered}
$$

where $i \cup j$ can be any disjoint decomposition of the set of indices $\{1, \ldots, n\}$ and $k, l=1, \ldots, i$. We remark that Legendre transformations are change of coordinates in $\mathscr{T}$ and that they are not defined in the equilibrium space. Moreover, the phase space $\mathscr{T}$ is equipped with a canonical contact structure called the Gibbs 1-form defined as

$$
\Theta=d \Phi-\delta_{a b} I^{b} d E^{a}=d \Phi-I_{a} d E^{a},
$$

which extremely resembles the first law of thermodynamics and hence will be the starting point to define the equilibrium space.

Furthermore, the equilibrium space $\mathscr{E}$ is the $n$-dimensional submanifold of $\mathscr{T}$ defined by the embedding $\varphi: \mathscr{E} \rightarrow \mathscr{T}$ under the condition

$$
\varphi^{*}(\Theta)=0, \quad \text { that is, } d \Phi=I_{a} d E^{a}, \quad I_{a}=\frac{\partial \Phi}{\partial E^{a}},
$$

where $\varphi^{*}$ is the pullback of $\varphi$. It follows immediately from (2) that (3) represents both the first law and the equilibrium conditions for the thermodynamic system under analysis, so that $\mathscr{E}$ results to be (by definition) the submanifold of points where the first law and the equilibrium conditions hold, that is, the geometric counterpart of the thermodynamic system. It also follows that the coordinates $\left\{Z^{A}\right\}$ of $\mathscr{T}$ assume a physical meaning in $\mathscr{E}$. In fact, the set $\left\{E^{a}\right\}$, with $a=1, \ldots, n$, can be identified with the extensive thermodynamic variables, while $\Phi=\Phi\left(E^{a}\right)$ with the fundamental equation for the thermodynamic potential, and finally the coordinates $\left\{I^{a}\right\}=$ $\left\{I^{a}\left(E^{a}\right)\right\} \equiv\left\{\partial_{E^{a}} \Phi\right\}, a=1, \ldots, n$ represent the intensive quantities corresponding to the extensive set $\left\{E^{a}\right\}$ (see, e.g., [48] for these definitions).

Now, let us add the Riemannian structure. Since we want the Riemannian structure to share the same properties of the first law and since the first law is invariant under Legendre transformations, we introduce in the phase space $\mathscr{T}$ a metric $G$ which is invariant under Legendre transformations. In GTD, there are several families of metrics which have this property (for a recent work on this topic see [49]). Among them, one has been proven particularly successful to describe systems with second-order phase transitions, as black holes are supposed to be. Thus, the candidate metric we shall use in this work is

$$
G=\left(d \Phi-I_{a} d E^{a}\right)^{2}+\Lambda\left(\xi_{a b} E^{a} I^{b}\right)\left(\chi_{c d} d E^{c} d I^{d}\right),
$$

where $\xi_{a b}$ and $\chi_{a b}$ are diagonal constant tensors and $\Lambda$ is an arbitrary Legendre invariant function of the coordinates $\left\{Z^{A}\right\}$. In particular, we choose to fix $\Lambda=1, \xi_{a b}=\delta_{a b} \equiv$ $\operatorname{diag}(1, \ldots, 1)$ and $\chi_{a b}=\eta_{a b} \equiv \operatorname{diag}(-1,1, \ldots, 1)$ in order to get the exact expression for the metric describing black holes phase transitions (see also [33]).

On the other side, we are not interested in the geometric representation of the phase space, while we care about the geometric properties of the thermodynamic system, which is paralleled by the equilibrium space $\mathscr{E}$. Thus, we pull back the metric $G$ onto $\mathscr{E}$ and obtain a Riemannian structure for the equilibrium space which reads

$$
g^{I I} \equiv \varphi^{*}(G)=\left(E^{a} \frac{\partial \Phi}{\partial E^{a}}\right)\left(\eta_{b}^{c} \frac{\partial^{2} \Phi}{\partial E^{c} \partial E^{d}} d E^{b} d E^{d}\right),
$$

where $\varphi^{*}$ is the pullback of $\varphi$ as in (3) and $\eta_{b}^{c}=$ $\operatorname{diag}(-1,1, \ldots, 1)$. We remark that $g^{I I}$ is (by definition) invariant under (total) Legendre transformations (see, e.g., [33]). Moreover, we also note that $g^{I I}$ can be calculated explicitly once the fundamental equation $\Phi=\Phi\left(E^{a}\right)$ is known.

The main thesis of GTD is that the thermodynamic properties of a system described by a fundamental equation $\Phi\left(E^{a}\right)$ can be translated into geometrical features of the equilibrium manifold $\mathscr{E}$, which in our case is described by the metric $g^{I I}$. For example, the scalar curvature of $\mathscr{E}$ should give information about the thermodynamic interaction. This means that systems without interaction shall correspond to flat geometries and systems showing interaction and phase transitions should correspond to curved equilibrium manifolds having curvature singularities. It has been tested in a number of works (see, e.g. [31-35]) that indeed such correspondence works. Furthermore, a previous work [41] studying the thermodynamics and GTD of the ReissnerNordström and of the Kerr black holes in any dimensions, highlighted that curvature singularities of $g^{I I}$ are exactly at the same points where the generalized susceptibilities diverge.

In this work, we extend the work in [41] to the case of Myers-Perry black holes in five dimensions, with the aim of both to analyze their thermodynamic geometry from a new perspective and to focus on the idea of checking what happens with a change of the potential from $\Phi=M$ to $\Phi=S$ in the GTD analysis and when the equilibrium manifold is 3 dimensional. The investigation of the phase structure of Myers-Perry black holes in five dimensions is thus a matter which is interesting by itself and that will provide us with the necessary ground for a new test of the correspondence between the thermodynamic geometry $g^{I I}$ of GTD and black holes thermodynamics.

\section{Myers-Perry Black Holes}

The Kerr black hole can be generalized to the case of arbitrary dimensions and arbitrary number of spins. It turns out that, provided, $d$ is the number of spacetime dimensions, that the maximum number of possible independent spins is $(d-1) / 2$ if $d$ is odd and $(d-2) / 2$ if $d$ is even [50]. Such general configurations are called Myers-Perry black holes. They deserve a special interest because they are the natural generalization of the well-known Kerr black hole to higher number of spins and because they are shown to coexist with the Emparan-Reall black ring solution for some values of the parameters, thus providing the first explicit example of a violation in a dimension higher than four of 
the uniqueness theorem (see, e.g., [51] for more details). The line element of the Myers-Perry black hole with an arbitrary number of independent angular momenta in BoyerLindquist coordinates for $d=2 n+1$ (i.e., odd $d$ ) reads [50]

$$
\begin{aligned}
d s^{2}= & -d t^{2}+\frac{\mu r^{2}}{\Pi F}\left(d t+\sum_{i=1}^{n} a_{i} \mu_{i}^{2} d \phi_{i}\right)^{2}+\frac{\Pi F}{\Pi-\mu r^{2}} d r^{2} \\
& +\sum_{i=1}^{n}\left(r^{2}+a_{i}^{2}\right)\left(d \mu_{i}^{2}+\mu_{i}^{2} d \phi_{i}^{2}\right)
\end{aligned}
$$

with

$$
\begin{aligned}
F \equiv 1-\sum_{i=1}^{n} \frac{a_{i}^{2} \mu_{i}^{2}}{r^{2}+a_{i}^{2}}, & \Pi \equiv \prod_{i=1}^{n}\left(r^{2}+a_{i}^{2}\right), \\
\mu \equiv \frac{16 \pi G M}{(d-2) \Omega_{(d-2)}}, & a_{i} \equiv \frac{(d-2)}{2} \frac{J_{i}}{M},
\end{aligned}
$$

where $\Omega_{(d-2)}=2 \pi^{n} / \Gamma(n), M$ is the mass of the black hole, $J_{i}=J_{1}, \ldots, J_{n}$ are the $(d-1) / 2$ independent angular momenta, and the constraint $\sum_{i=1}^{n} \mu_{i}^{2}=1$ holds. Solving the equation $g^{r r}=1 / g_{r r}=0$, one finds the radius of the event horizon (in any dimensions) and thus derives the area and the corresponding entropy, using Bekenstein area law [29].

In particular, in this work, we are interested in the five dimensional case, that is, when $d=5$. Myers-Perry black holes in five-dimensions can have up to 2 independent angular momenta, and the general equation for the area reads [29]

$$
A=\frac{2 \pi^{2}}{r_{+}}\left(r_{+}^{2}+a_{1}^{2}\right)\left(r_{+}^{2}+a_{2}^{2}\right)
$$

where $r_{+}$is the radius of the event horizon. From the previous expression the entropy can be calculated, being

$$
S=\frac{k_{B} A}{4 G}=\frac{1}{r_{+}}\left(r_{+}^{2}+a_{1}^{2}\right)\left(r_{+}^{2}+a_{2}^{2}\right),
$$

where we choose $k_{B}$ and $G$ such that $S$ simplifies as in the second equality in (9).

Since it is rather complicated to calculate explicitly the previous expression for the entropy, we will use the $M$ representation throughout the paper. This is possible since the mass can be written in terms of $S, J_{1}$, and $J_{2}$ as [29]

$$
M\left(S, J_{1}, J_{2}\right)=\frac{3}{4} S^{2 / 3}\left(1+4 \frac{J_{1}^{2}}{S^{2}}\right)^{1 / 3}\left(1+4 \frac{J_{2}^{2}}{S^{2}}\right)^{1 / 3}
$$

Equation (10) thus represents the fundamental equation for the Myers-Perry black hole in five dimensions as a thermodynamic system. Starting from (10), we can analyze both the thermodynamic properties and their geometrothermodynamic counterparts. We will split the work in order to consider the three most interesting cases, that is, when one of the two angular momenta is zero, when they are both nonzero but equal, and finally when they are both nonzero and different among each other.
3.1. The Case $J_{2}=0$. If either $J_{1}=0$ or $J_{2}=0$, we obtain the Kerr black hole in 5 dimensions, which has been analyzed in [41]. We briefly review here some of the results presented there and improve the analysis, including the investigation of the response functions defined in the total Legendre transformation of the mass $M$, which we will call the Gibbsian response functions, in analogy with standard thermodynamics [48]. Therefore, let us suppose that $J_{2}=$ 0 . According to our previous results [41], we know that the response functions defined in the mass representation read

$$
\begin{gathered}
C_{J_{1}}=-\frac{3 S\left(S^{2}+4 J_{1}^{2}\right)}{S^{2}-12 J_{1}^{2}}, \quad \kappa_{S}=\frac{3\left(S^{2}+4 J_{1}^{2}\right)^{5 / 3}}{2 J_{1}\left(3 S^{2}-4 J_{1}^{2}\right)}, \\
\alpha_{S}=-\frac{3}{8} \frac{\left(S^{2}+4 J_{1}^{2}\right)^{5 / 3}}{J_{1}^{2} S},
\end{gathered}
$$

where we make use of the notation $M_{E^{a}} \equiv \partial M / \partial E^{a}$ and $M_{E^{a} E^{b}} \equiv \partial^{2} M / \partial E^{a} \partial E^{b}$, for $E^{i}=S, J_{1}$. It follows that $\alpha_{S}$ does not show any singularity (apart from the extremal limit $S=0$ ), while $C_{J_{1}}$ diverges at the Davies point $S^{2}=$ $12 J_{1}^{2}$ and $\kappa_{S}$ shows an additional possible phase transition at $3 S^{2}=4 J_{1}^{2}$. As it was pointed out in [41], both singularities of the heat capacity and of the compressibility are in the black hole region and hence are physically relevant. It was also shown that the GTD geometry (5) with fundamental equation (10) (with $J_{2}=0$ ) is curved, indicating the presence of thermodynamic interaction, and that the singularities of the scalar curvature are situated exactly at the same points where the response functions $C_{J_{1}}$, and $\kappa_{S}$ diverge, both in the mass and in the entropy representations. Furthermore, it was also commented that Weinhold geometry is flat in this case and Ruppeiner thermodynamic metric diverges only in the extremal limit $S=0$ (see, e.g., [29] for a complete analysis using these metrics).

Moreover, since the thermodynamics of black holes can depend on the chosen ensemble (see, e.g., $[52,53]$ ), we now proceed to calculate the Gibbsian response functions, which can possibly give new information about the phase structure. Using the relations between thermodynamic derivatives (see, [48]), we find out that the expressions for such response functions in the coordinates $\left(S, J_{1}\right)$ used here are

$$
\begin{gathered}
C_{\Omega_{1}}=-\frac{S\left(3 S^{2}-4 J_{1}^{2}\right)}{S^{2}+4 J_{1}^{2}}, \quad \kappa_{T}=-\frac{S^{2}-12 J_{1}^{2}}{2 J_{1}\left(S^{2}+4 J_{1}^{2}\right)^{1 / 3}}, \\
\alpha_{\Omega_{1}}=-\frac{8 S}{\left(S^{2}+4 J_{1}^{2}\right)^{1 / 3}} .
\end{gathered}
$$

It is immediate to see that $C_{\Omega_{1}}$ never diverges and it vanishes exactly at the same points where $\kappa_{S}$ diverges. On the other side, $\kappa_{T}$ is never divergent and it vanishes exactly where $C_{J_{1}}$ diverges, while $\alpha_{\Omega_{1}}$ is always finite. It follows that the Gibbsian response functions do not add any information to the knowledge of the phase structure of this configuration, as they change the sign exactly at the points that we have already analyzed; therefore, we conclude that the divergences of the 
scalar curvature of the metric (5) match exactly the points of second-order phase transitions.

Let us now add a second spin parameter and show that there is still a concrete correlation between the geometric description performed with $g^{I I}$ and the thermodynamic properties. To do so, we first focus on the special case of (10) in which $J_{1}=J_{2}=J$, and afterwards we will consider the completely general case, that is, with $J_{1}$ and $J_{2}$ both different from zero and from each other. In particular, in the latter case, we will get a 3-dimensional thermodynamic manifold labelled by $\left(E^{1}=S, E^{2}=J_{1}, E^{3}=J_{2}\right)$, and hence we will consider the 3 -dimensional version of the metric (5).

3.2. The Case $J_{1}=J_{2} \equiv J$. Another special case in (10) which is of interest is the case in which the two angular momenta are fixed to be equal, that is, $J_{1}=J_{2} \equiv J$. This is interesting from the mathematical and physical point of view since it is the only case in which the angular momenta are both different from zero, and at the same time the thermodynamic manifold is 2-dimensional. In fact, the mass fundamental equation (10) in this case is given by

$$
M(S, J)=\frac{3}{4} S^{2 / 3}\left(1+4 \frac{J^{2}}{S^{2}}\right)^{2 / 3},
$$

and the response functions can then be accordingly calculated to give

$$
\begin{gathered}
C_{J}=-\frac{3 S\left(S^{4}-16 J^{4}\right)}{S^{4}-32 J^{2} S^{2}-80 J^{4}}, \quad \kappa_{S}=\frac{3 S^{2 / 3}\left(S^{2}+4 J^{2}\right)^{4 / 3}}{4 J\left(3 S^{2}+4 J^{2}\right)}, \\
\alpha_{S}=-\frac{3}{16} \frac{S^{5 / 3}\left(S^{2}+4 J^{2}\right)^{4 / 3}}{J^{2}\left(S^{2}+2 J^{2}\right)} .
\end{gathered}
$$

From (14), it follows that in this case $\alpha_{S}$ and $\kappa_{S}$ do not show any singularity, while $C_{J}$ diverges at the roots of the denominator $\mathscr{D}_{C}=S^{4}-32 J^{2} S^{2}-80 J^{4}$. We also observe that the temperature of this black hole is given by

$$
T \equiv\left(\frac{\partial M}{\partial S}\right)_{J}=\frac{1}{2} \frac{S^{2}-4 J^{2}}{S^{5 / 3}\left(S^{2}+4 J^{2}\right)^{1 / 3}},
$$

therefore, the extremal limit $T=0$ is reached when $J^{2} / S^{2}=$ $1 / 4$.

Solving $\mathscr{D}_{C}=0$, we find that the singularities of the heat capacity are situated at a value $S_{\text {critical }}$ for the entropy such that

$$
\left.\frac{J^{2}}{S^{2}}\right|_{S=S_{\text {critical }}}=\frac{\sqrt{21}-4}{20},
$$

which is less than the extremal limit. Therefore Davies point of phase transition belongs to the black hole region and we shall investigate it.

It is convenient also in this case to write the full set of thermodynamic response functions, including the Gibbsian ones.
Again, making use of the relations between thermodynamic derivatives, we find out that they read

$$
\begin{gathered}
C_{\Omega}=-\frac{S\left(S^{2}-4 J^{2}\right)\left(3 S^{2}+4 J^{2}\right)}{\left(S^{2}+4 J^{2}\right)^{2}}, \\
\kappa_{T}=-\frac{S^{2 / 3}}{4 J} \frac{\mathscr{D}_{C}}{\left(S^{2}+4 J^{2}\right)^{5 / 3}}, \\
\alpha_{\Omega}=\frac{8 S^{5 / 3}\left(S^{2}+2 J^{2}\right)}{\left(S^{2}+4 J^{2}\right)^{5 / 3}} .
\end{gathered}
$$

In this case, we observe that the only divergence of the response functions in (14), that is, the divergence of $C_{J}$, is again controlled by the vanishing of $\kappa_{T}$. Furthermore, both $C_{J}$ and $C_{\Omega}$ vanish at the extremal limit, but this does not correspond to any divergence of $\kappa_{S}$, and hence we expect the curvature of the thermodynamic metric to diverge only at the points where $\mathscr{D}_{C}=0$.

From the point of view of Geometrothermodynamics, given the fundamental equation (13) and the general metric (5), we can calculate the particular metric and the scalar curvature for the equilibrium manifold of the MP black hole with two equal angular momenta, both in the mass and in the entropy representations.

The metric in the $M$ representation reads

$$
\begin{aligned}
g_{M}^{I I}= & \frac{1}{S^{4 / 3}\left(S^{2}+4 J^{2}\right)^{2 / 3}} \\
& \times\left\{\frac{1}{12} \frac{\mathscr{D}_{C}}{S^{2}} d S^{2}+\frac{2\left(3 S^{2}+4 J^{2}\right)}{3} d J^{2}\right\} .
\end{aligned}
$$

Therefore, its scalar curvature is

$$
\begin{aligned}
R_{M}= & 24 S^{10 / 3}\left(S^{2}+4 J^{2}\right)^{2 / 3} \\
& \times\left(5 S^{6}+48 J^{2} S^{4}-368 J^{4} S^{2}-896 J^{6}\right) \\
& \times\left(\mathscr{D}_{C}^{2}\left(3 S^{2}+4 J^{2}\right)^{2}\right)^{-1} .
\end{aligned}
$$

The numerator is a not very illuminating function that never vanishes when the denominator is zero, and $\mathscr{D}_{C}$ is exactly the denominator of the heat capacity $C_{J}$. Therefore, the singularities of $R_{M}$ correspond exactly to those of $C_{J}$ (resp., to the vanishing of $\kappa_{T}$ ). We remark that the factor $3 S^{2}+4 J^{2}$ in the denominator of $R_{M}$, despite being always different from 0 (thus not indicating any phase transition in this case), is exactly the denominator of the compressibility $\kappa_{S}$ (resp., a factor in the numerator of $C_{\Omega}$ ).

To continue with the analysis, in [49], a general relation was presented (see (34) therein) to express $g^{I I}$ with $\Phi=S$ (i.e., in the $S$ representation) in the coordinates of the $M$ 
representation (i.e., $\left.\left\{E^{a}\right\}=(S, J)\right)$. Such relation in the present case reads

$$
\begin{aligned}
g_{S}^{I I}= & \frac{M-J \Omega}{T^{3}} \\
\times & {\left[T M_{S S} d S^{2}+2 \Omega M_{S S} d S d J\right.} \\
& \left.+\left(2 \Omega M_{S J}-T M_{J J}\right) d J^{2}\right],
\end{aligned}
$$

where $T \equiv \partial M / \partial S$ is the temperature, $\Omega \equiv \partial M / \partial J$ is the angular velocity at the horizon and $M_{E^{a} E^{b}} \equiv \partial^{2} M / \partial E^{a} \partial E^{b}$, for $E^{i}=S, J$. Using (20) and (13) for the mass in terms of $S$ and $J$, we can calculate the expression for metric $g_{S}^{I I}$ in the coordinates $(S, J)$, which reads

$$
\begin{aligned}
g_{S}^{I I}= & \frac{1}{3\left(S^{2}+4 J^{2}\right)(S+2 J)^{2}(S-2 J)^{2}} \\
& \times\left\{-\frac{3 S^{2}-4 J^{2}}{2} \mathscr{D}_{C} d S^{2}+\right. \\
& +\frac{8 S J\left(3 S^{2}-4 J^{2}\right)}{(S+2 J)(S-2 J)} \mathscr{D}_{C} d S d J-4 S^{2} \\
& \left.\quad \times \frac{9 S^{6}+156 S^{4} J^{2}+112 S^{2} J^{4}-448 J^{6}}{(S+2 J)(S-2 J)} d J^{2}\right\} .
\end{aligned}
$$

Consequently, the scalar curvature is

$$
R_{S}=\frac{\mathcal{N}_{S}}{\left(3 S^{2}-4 J^{2}\right)^{3}\left(S^{2}+4 J^{2}\right)^{2} \mathscr{D}_{C}^{2}},
$$

where $\mathcal{N}_{S}$ is again a function which never vanishes at the points where the denominator is zero. From (22), we see that the denominator of $C_{J}$ is present in the denominator of $R_{S}$. Furthermore, the factor $S^{2}+4 J^{2}$ is never zero, and hence it does not give any additional singularity. On the other hand, the factor $3 S^{2}-4 J^{2}$ is clearly vanishing when $J^{2} / S^{2}=3 / 4$, which is readily greater than the extremal limit $J^{2} / S^{2}=1 / 4$, and hence it has no physical relevance in our analysis.

We thus conclude that also in this case the GTD geometry $g^{I I}$ exactly reproduces the phase transition structure of the Myers-Perry black holes both in the mass and in the entropy representation. We comment that in the entropy representation there is an additional singularity which does not correspond to any singularity of the response functions. However, such singularity is situated out of the black hole region, and thus it is not to be considered here. We also remark that Ruppeiner curvature in this case reads $R=$ $-S\left(S^{2}+12 J^{2}\right) /\left(S^{4}-16 J^{4}\right)$, and hence it diverges only in the extremal limit, while Weinhold metric is flat.

In the next subsection, we will analyze the general case of the Myers-Perry black hole in five dimensions, that is, when the two angular momenta are allowed to vary freely.

3.3. The General Case in Which $J_{1} \neq J_{2} \neq 0$. Perhaps the most interesting case is the most general one, in which the two angular momenta are allowed to vary freely. In this case, the thermodynamic manifold is 3 dimensional and the mass fundamental equation is given by (10).

The generalized susceptibilities can then be accordingly calculated. The heat capacity at constant angular momenta $J_{1}$ and $J_{2}$ reads

$$
\begin{aligned}
C_{J_{1}, J_{2}} & =\frac{M_{S}}{M_{S S}} \\
& =-\frac{3 S\left(S^{2}+4 J_{1}^{2}\right)\left(S^{2}+4 J_{2}^{2}\right)\left(S^{4}-16 J_{1}^{2} J_{2}^{2}\right)}{\mathscr{D}_{C}},
\end{aligned}
$$

where

$$
\begin{aligned}
\mathscr{D}_{C}= & S^{8}-12\left(J_{1}^{2}+J_{2}^{2}\right) S^{6}-320 J_{1}^{2} J_{2}^{2} S^{4} \\
& -576 J_{1}^{2} J_{2}^{2}\left(J_{1}^{2}+J_{2}^{2}\right) S^{2}-1280 J_{1}^{4} J_{2}^{4} .
\end{aligned}
$$

Furthermore, one can define the 3 analogues of the adiabatic compressibility as

$$
\begin{aligned}
\left(\kappa_{S}\right)_{11} & \equiv\left(\frac{\partial J_{1}}{\partial \Omega_{1}}\right)_{S} \\
& =\frac{3 S^{2 / 3}\left(S^{2}+4 J_{1}^{2}\right)^{5 / 3}}{2\left(S^{2}+4 J_{2}^{2}\right)^{1 / 3}\left(3 S^{2}-4 J_{1}^{2}\right)}, \\
\left(\kappa_{S}\right)_{22} & \equiv\left(\frac{\partial J_{2}}{\partial \Omega_{2}}\right)_{S} \\
& =\frac{3 S^{2 / 3}\left(S^{2}+4 J_{2}^{2}\right)^{5 / 3}}{2\left(S^{2}+4 J_{1}^{2}\right)^{1 / 3}\left(3 S^{2}-4 J_{2}^{2}\right)}, \\
\left(\kappa_{S}\right)_{12} & \equiv\left(\frac{\partial J_{1}}{\partial \Omega_{2}}\right)_{S} \\
& =\frac{3}{16} \frac{S^{2 / 3}\left(S^{2}+4 J_{1}^{2}\right)^{2 / 3}\left(S^{2}+4 J_{2}^{2}\right)^{2 / 3}}{J_{1} J_{2}} .
\end{aligned}
$$

Finally, the analogues of the expansion are given by

$$
\begin{aligned}
\alpha_{S, J_{2}} & \equiv\left(\frac{\partial J_{1}}{\partial T}\right)_{S} \\
& =-\frac{3}{8} \frac{S^{5 / 3}\left(S^{2}+4 J_{1}^{2}\right)^{5 / 3}\left(S^{2}+4 J_{2}^{2}\right)^{2 / 3}}{J_{1}\left(S^{4}+6 S^{2} J_{2}^{2}+8 J_{1}^{2} J_{2}^{2}\right)}, \\
\alpha_{S, J_{1}} & \equiv\left(\frac{\partial J_{1}}{\partial T}\right)_{S} \\
& =-\frac{3}{8} \frac{S^{5 / 3}\left(S^{2}+4 J_{2}^{2}\right)^{5 / 3}\left(S^{2}+4 J_{1}^{2}\right)^{2 / 3}}{J_{2}\left(S^{4}+6 S^{2} J_{1}^{2}+8 J_{1}^{2} J_{2}^{2}\right)} .
\end{aligned}
$$

In this case, neither $\left(\kappa_{S}\right)_{12}$ nor the expansions show any singularity, while $C_{J_{1}, J_{2}}$ diverges when $\mathscr{D}_{C}=0$ and the compressibilities $\left(\kappa_{S}\right)_{11}$ and $\left(\kappa_{S}\right)_{22}$ diverge when $3 S^{2}-4 J_{1}^{2}=0$ 


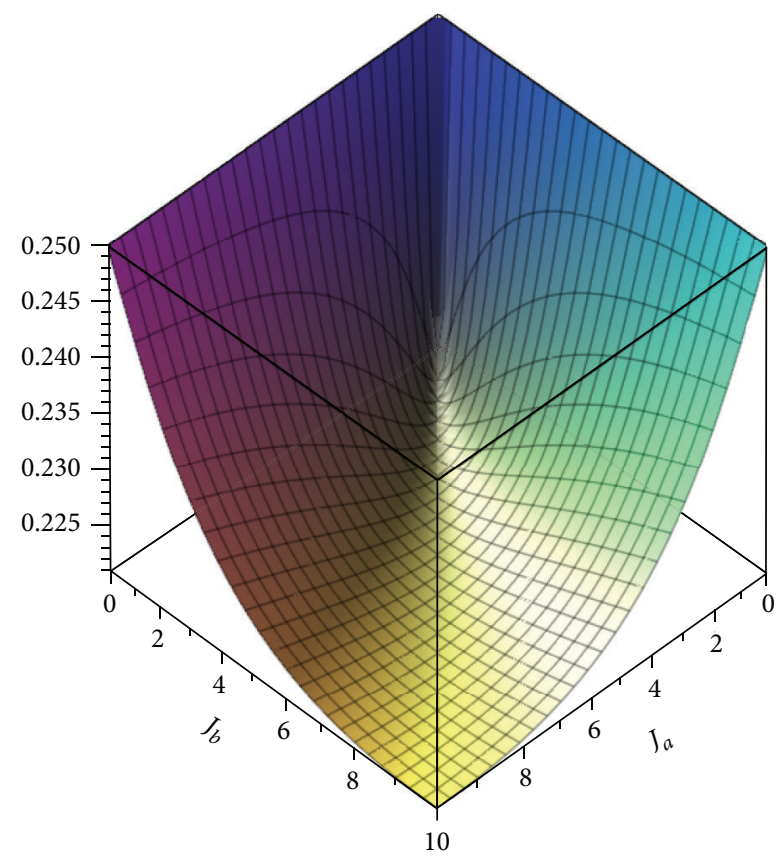

FIGURE 1: The difference between the extremal limit $\left(J_{1} J_{2} / S^{2}=1 / 4\right)$ and the value of $J_{1} J_{2} / S^{2}$ at the critical point of the heat capacity, plotted for values of $J_{1}$ and $J_{2}$ in the interval $[0,10]$.

and $3 S^{2}-4 J_{2}^{2}=0$, respectively. Furthermore, the temperature reads

$$
T=\frac{1}{2 S^{5 / 3}} \frac{S^{4}-16 J_{1}^{2} J_{2}^{2}}{\left(S^{2}+4 J_{1}^{2}\right)^{2 / 3}\left(S^{2}+4 J_{2}^{2}\right)^{2 / 3}} .
$$

Hence; the extremal limit is reached for $J_{1} J_{2} / S^{2}=1 / 4$. The heat capacity diverges when $\mathscr{D}_{C}=0$, which is an algebraic equation of degree 8 in $S$. We can solve numerically such equation and obtain the critical value $S=\mathrm{S}_{\text {critical }}$ in terms of $J_{1}$ and $J_{2}$. Taking only the roots which are real and positive, we can compare them with the extremal limit by doing

$$
\left.\frac{J_{1} J_{2}}{S^{2}}\right|_{S=\text { Sextremal }}-\left.\frac{J_{1} J_{2}}{S^{2}}\right|_{S=S \text { Scritical }}=\frac{1}{4}-\left.\frac{J_{1} J_{2}}{S^{2}}\right|_{S=\text { Scritical }} .
$$

The plot of the result is given in Figure 1 for some values of $J_{1}$ and $J_{2}$. As we can see from Figure 1, the difference in (28) is always positive, and hence the point of phase transition signaled by the divergence of the heat capacity is always in the black hole region.

In the same way, we can solve $3 S^{2}-4 J_{1}^{2}=0$ and see whether the divergence of $\left(\kappa_{S}\right)_{11}$ lies in the black hole region or not. It turns out that the denominator of $\left(\kappa_{S}\right)_{11}$ vanishes for values of $S$ such that $J_{1}^{2} / S^{2}=3 / 4$, which means that $J_{1} J_{2} / S^{2}=$ $(3 / 4)\left(J_{2} / J_{1}\right)$. Therefore, we have that $(1 / 4)-(3 / 4)\left(J_{2} / J_{1}\right)$ is positive provided that $J_{1}>3 J_{2}$ for $J_{1}>0$ or $J_{1}<3 J_{2}$ for $J_{1}<0$. Summing up, the divergences of $\left(\kappa_{S}\right)_{11}$ can be in the black hole region for appropriate values of $J_{1}$ and $J_{2}$. Analogously, the divergences of $\left(\kappa_{S}\right)_{22}$ can also be in the black hole region.

As in the preceding sections, we will now focus on the Gibbsian response functions, in order to make the analysis complete. The heat capacity at constant angular velocities read

$$
\begin{aligned}
C_{\Omega_{1}, \Omega_{2}} \equiv & T\left(\frac{\partial S}{\partial T}\right)_{\Omega_{1}, \Omega_{2}} \\
= & -3 S\left(S^{4}-16 J_{1}^{2} J_{2}^{2}\right)\left(3 S^{2}-4 J_{1}^{2}\right) \\
& \times\left(3 S^{2}-4 J_{2}^{2}\right)\left(S^{2}+4 J_{1}^{2}\right)\left(S^{2}+4 J_{2}^{2}\right) \\
& \times\left(\mathscr{D}\left(S, J_{1}, J_{2}\right)\right)^{-1},
\end{aligned}
$$

where the denominator is given by

$$
\begin{aligned}
\mathscr{D}\left(S, J_{1}, J_{2}\right)= & 9 S^{12}+72\left(J_{1}^{2}+J_{2}^{2}\right) S^{10} \\
& +16\left(9 J_{1}^{4}+95 J_{1}^{2} J_{2}^{2}+9 J_{2}^{4}\right) S^{8} \\
& +5376 J_{1}^{2} J_{2}^{2}\left(J_{1}^{2}+J_{2}^{2}\right) S^{6} \\
& -256 J_{1}^{2} J_{2}^{2}\left(9 J_{1}^{4}-101 J_{1}^{2} J_{2}^{2}+9 J_{2}^{4}\right) S^{4} \\
& -6144 J_{1}^{4} J_{2}^{4}\left(J_{1}^{2}+J_{2}^{2}\right) S^{2}-53248 J_{1}^{6} J_{2}^{6} .
\end{aligned}
$$

Furthermore, one can define three generalized susceptibilities, analogous to the isothermal compressibility, as

$$
\begin{gathered}
\left(\kappa_{T}\right)_{11} \equiv\left(\frac{\partial J_{1}}{\partial \Omega_{1}}\right)_{T}, \quad\left(\kappa_{T}\right)_{12} \equiv\left(\frac{\partial J_{1}}{\partial \Omega_{2}}\right)_{T}, \\
\left(\kappa_{T}\right)_{22} \equiv\left(\frac{\partial J_{2}}{\partial \Omega_{2}}\right)_{T} .
\end{gathered}
$$

For the Myers-Perry black hole it can be written as

$$
\begin{aligned}
& \left(\kappa_{T}\right)_{11} \\
& =-\frac{S^{2 / 3}}{2}\left(\mathscr { D } _ { C } \left(\left(S^{2}+4 J_{1}^{2}\right)^{1 / 3}\left(S^{2}+4 J_{2}^{2}\right)^{1 / 3}\right.\right. \\
& \left.\left.\times\left(S^{6}-12 J_{2}^{2} S^{4}+48 J_{1}^{2} J_{2}^{2} S^{2}+192 J_{2}^{2} J_{2}^{4}\right)\right)^{-1}\right), \\
& \left(\kappa_{T}\right)_{22} \\
& =-\frac{S^{2 / 3}}{2}\left(\mathscr { D } _ { C } \left(\left(S^{2}+4 J_{1}^{2}\right)^{1 / 3}\left(S^{2}+4 J_{2}^{2}\right)^{1 / 3}\right.\right. \\
& \left.\left.\times\left(S^{6}-12 J_{1}^{2} S^{4}+48 J_{1}^{2} J_{2}^{2} S^{2}+192 J_{2}^{2} J_{1}^{4}\right)\right)^{-1}\right),
\end{aligned}
$$

while $\left(\kappa_{T}\right)_{12}$ has a more cumbersome expression and we will not write it here, since it has the same properties of $\left(\kappa_{T}\right)_{11}$ and $\left(\kappa_{T}\right)_{22}$ as regards to our analysis; that is, it is proportional to the denominator of $C_{J_{1}, J_{2}}$ defined in (24) and it has a nontrivial denominator (one can also introduce the two analogues of the thermal expansion, but for the sake of simplicity, we are not going to write them here, since they do not show any singularities, and hence they do not play any role in our analysis). 
Therefore, from the thermodynamic point of view, we remark that the divergences of $C_{J_{1}, J_{2}}$ are matched by the vanishing of the three quantities $\left(\kappa_{T}\right)_{i j}$, while the divergences of $\left(\kappa_{S}\right)_{11}$ and $\left(\kappa_{S}\right)_{22}$ are reproduced as zeroes of the heat capacity $C_{\Omega_{1}, \Omega_{2}}$. This behavior is in agreement with the analysis of the preceding sections. Furthermore, in this case, the heat capacity $C_{\Omega_{1}, \Omega_{2}}$ and the generalized compressibilities $\left(\kappa_{T}\right)_{i j}$ possibly show additional phase transitions, which is a further indication of the fact that black holes exhibit different thermodynamic behavior in different potentials.

Now, let us turn to the GTD analysis. Given the fundamental equation (10) and the general metric (5), we can calculate the particular metric and the scalar curvature for the MP black hole with two free angular momenta, both in the mass and in the entropy representations. The metric in the $M$ representation reads

$$
\begin{aligned}
g_{M}^{I I}= & \frac{1}{3 S^{4 / 3}\left(S^{2}+4 J_{1}^{2}\right)^{1 / 3}\left(S^{2}+4 J_{2}^{2}\right)^{1 / 3}} \\
& \times\left\{-\frac{1}{4} \frac{\mathscr{D}_{C}}{S^{2}\left(S^{2}+4 J_{1}^{2}\right)\left(S^{2}+4 J_{2}^{2}\right)} d S^{2}\right. \\
& +\frac{\left(3 S^{2}-4 J_{1}^{2}\right)\left(S^{2}+4 J_{2}^{2}\right)}{S^{2}+4 J_{1}^{2}} d J_{1}^{2} \\
& +\frac{\left(3 S^{2}-4 J_{2}^{2}\right)\left(S^{2}+4 J_{1}^{2}\right)}{S^{2}+4 J_{2}^{2}} d J_{2}^{2} \\
& \left.+16 J_{1} J_{2} d J_{1} d J_{2}\right\} .
\end{aligned}
$$

Hence, its scalar curvature is

$$
\begin{aligned}
R_{M}= & \mathcal{N}_{M} \\
\times & \left(\mathscr{D}_{C}^{2}\left[3 S^{4}-4 S^{2}\left(J_{1}^{2}+J_{2}^{2}\right)-16 J_{1}^{2} J_{2}^{2}\right]^{2}\right. \\
& \left.\times\left(S^{2}+4 J_{1}^{2}\right)^{2 / 3}\left(S^{2}+4 J_{2}^{2}\right)^{2 / 3}\right)^{-1},
\end{aligned}
$$

where $\mathscr{D}_{C}$ is as usual the denominator of $C_{J_{1}, J_{2}}$ defined in (24). Since there is no term in the numerator $\mathcal{N}_{M}$ which cancels out the divergences that happen when $\mathscr{D}_{C}=0$, we can conclude that every phase transition related to the heat capacity $C_{J_{1}, J_{2}}$ is properly reproduced by the scalar curvature $R_{M}$. In addition, in this case, the factor $3 S^{4}-4 S^{2}\left(J_{1}^{2}+\right.$ $\left.J_{2}^{2}\right)-16 J_{1}^{2} J_{2}^{2}$ can also vanish, possibly giving an additional singularity which does not correspond to the ones shown by the response functions. It is easy to calculate that $3 S^{4}-4 S^{2}\left(J_{1}^{2}+\right.$ $\left.J_{2}^{2}\right)-16 J_{1}^{2} J_{2}^{2}=0$ for values of $S$ such that

$$
\frac{J_{1} J_{2}}{S^{2}}=\frac{1}{8} \frac{\left(-J_{1}^{2}-J_{2}^{2}+\sqrt{J_{1}^{4}+14 J_{1}^{2} J_{2}^{2}+J_{2}^{4}}\right)}{J_{1} J_{2}} .
$$

We can thus calculate the difference between the extremal limit $J_{1} J_{2} / S^{2}=1 / 4$ and the critical value (35). The result is

$$
\begin{aligned}
& \frac{1}{4}-\frac{1}{8} \frac{\left(-J_{1}^{2}-J_{2}^{2}+\sqrt{J_{1}^{4}+14 J_{1}^{2} J_{2}^{2}+J_{2}^{4}}\right)}{J_{1} J_{2}} \\
& =-\frac{1}{48}\left(\left(\left(J_{1}^{2}+J_{2}^{2}-6 J_{1} J_{2}+\sqrt{J_{1}^{4}+14 J_{1}^{2} J_{2}^{2}+J_{2}^{4}}\right)\right.\right. \\
& \left.\times\left(J_{1}^{2}+J_{2}^{2}-\sqrt{J_{1}^{4}+14 J_{1}^{2} J_{2}^{2}+J_{2}^{4}}\right)\right) \\
& \left.\times\left(J_{1}^{2} J_{2}^{2}\right)^{-1}\right),
\end{aligned}
$$

which can be positive for appropriate values of $J_{1}$ and $J_{2}$. Therefore, such points of divergence of $R_{M}$ are in the black hole region for some values of the parameters. Hence, we conclude that the behavior of $R_{M}$ perfectly matches the behavior of $C_{J_{1}, J_{2}}$, but in this case, it does not reproduce the additional possible phase transitions indicated by the singularities of the compressibilities $\left(\kappa_{S}\right)_{11}$ and $\left(\kappa_{S}\right)_{22}$ and possibly shows some additional unexpected singularities.

However, we can give a precise physical meaning to such additional singularities. In fact, if we evaluate the determinant of the Hessian of the mass with respect to the angular momenta $J_{1}$ and $J_{2}$, we get

$$
\begin{aligned}
\operatorname{det}\left(\operatorname{Hess}(M)_{J_{1} J_{2}}\right) & \equiv M_{J_{1_{1} J_{1}}} M_{J_{2} J_{2}}-M_{J_{1} J_{2}}^{2} \\
& =\frac{4}{3} \frac{3 S^{4}-4 S^{2}\left(J_{1}^{2}+J_{2}^{2}\right)-16 J_{1}^{2} J_{2}^{2}}{S^{4 / 3}\left(S^{2}+4 J_{1}^{2}\right)^{4 / 3}\left(S^{2}+4 J_{2}^{2}\right)^{4 / 3}},
\end{aligned}
$$

from which we can see that the numerator is exactly the factor in the denominator of $R_{M}$ whose roots give the additional singularities. Since the Hessians of the energy in thermodynamics are related to the stability conditions, we suggest that the physical meaning of such additional divergences of $R_{M}$ is to be found in a change of stability of the system, for example, from a stable phase to an unstable one.

On the other side, using the relation (20) for $g^{I I}$ between the $M$ and the $S$ representations, naturally extended to the 3 -dimensional case with coordinates $\left(S, J_{1}, J_{2}\right)$, that is,

$$
\begin{aligned}
g_{S}^{I I}= & \frac{M-J_{1} \Omega_{1}-J_{2} \Omega_{2}}{T^{3}} \\
\times[ & T M_{S S} d S^{2}+2 \Omega_{1} M_{S S} d S d J_{1}+2 \Omega_{2} M_{S S} d S d J_{2} \\
& +\left(2 \Omega_{1} M_{S J_{1}}-T M_{J_{1} J_{1}}\right) d J_{1}^{2} \\
& +\left(2 \Omega_{2} M_{S J_{2}}-T M_{J_{2} J_{2}}\right) d J_{2}^{2} \\
& \left.-2\left(T M_{J_{1} J_{2}}-\Omega_{1} M_{S J_{2}}-\Omega_{2} M_{S J_{1}}\right) d J_{1} d J_{2}\right],
\end{aligned}
$$


we can now calculate the metric in the $S$ representation, which reads

$$
\begin{aligned}
& g_{S}^{I I}=\frac{\left[3 S^{4}+4 S^{2}\left(J_{1}^{2}+J_{2}^{2}\right)-16 J_{1}^{2} J_{2}^{2}\right] \mathscr{D}_{C}}{3\left(S^{2}+4 J_{1}^{2}\right)\left(S^{2}+4 J_{2}^{2}\right)\left(S^{2}-4 J_{1} J_{2}\right)^{2}\left(S^{2}+4 J_{1} J_{2}\right)^{2}} \\
& \times\left\{\frac{1}{2} d S^{2}+4 \frac{S J_{1}\left(S^{2}+4 J_{2}^{2}\right)}{\left(S^{2}-4 J_{1} J_{2}\right)\left(S^{2}+4 J_{1} J_{2}\right)} d S d J_{1}\right. \\
& +4 \frac{S J_{2}\left(S^{2}+4 J_{1}^{2}\right)}{\left(S^{2}-4 J_{1} J_{2}\right)\left(S^{2}+4 J_{1} J_{2}\right)} d S d J_{2} \\
& -2\left(S^{2}\left(S^{2}+4 J_{2}^{2}\right)^{2}\right. \\
& \times\left[3 S^{6}+26 S^{4} J_{1}^{2}+144 S^{2} J_{1}^{2} J_{2}^{2}+320 J_{1}^{4} J_{2}^{2}\right] \\
& \left.\times\left(\mathscr{D}_{C}\left(S^{2}-4 J_{1} J_{2}\right)\left(S^{2}+4 J_{1} J_{2}\right)\right)^{-1}\right) d J_{1}^{2} \\
& -2\left(S^{2}\left(S^{2}+4 J_{1}^{2}\right)^{2}\right. \\
& \times\left[3 S^{6}+26 S^{4} J_{2}^{2}+144 S^{2} J_{1}^{2} J_{2}^{2}+320 J_{2}^{4} J_{1}^{2}\right] \\
& \left.\times\left(\mathscr{D}_{C}\left(S^{2}-4 J_{1} J_{2}\right)\left(S^{2}+4 J_{1} J_{2}\right)\right)^{-1}\right) d J_{2}^{2} \\
& -32\left(S^{2} J_{1} J_{2}\left(S^{2}+4 J_{1}^{2}\right)\left(S^{2}+4 J_{2}^{2}\right)\right. \\
& \times\left[5 S^{4}+12 S^{2}\left(J_{1}^{2}+J_{2}^{2}\right)+16 J_{1}^{2} J_{2}^{2}\right] \\
& \times\left(\mathscr{D}_{C}\left(S^{2}-4 J_{1} J_{2}\right)\right. \\
& \left.\left.\left.\left.\times\left(S^{2}+4 J_{1} J_{2}\right)\right)^{-1}\right)\right) d J_{1} d J_{2}\right\} .
\end{aligned}
$$

The scalar curvature can thus be calculated to obtain

$$
\begin{aligned}
R_{S}=\mathcal{N}_{S} \times( & \mathscr{D}_{C}^{2}\left[3 S^{4}+4 S^{2}\left(J_{1}^{2}+J_{2}^{2}\right)-16 J_{1}^{2} J_{2}^{2}\right]^{3} \\
& \left.\times S^{2}\left(S^{2}+4 J_{1}^{2}\right)\left(S^{2}+4 J_{2}^{2}\right)\right)^{-1} .
\end{aligned}
$$

In this case, we see again that the denominator of the heat capacity $\mathscr{D}_{C}$ is present in the denominator of $R_{S}$. Furthermore, the second factor, which is slightly different from the factor in the denominator of $R_{M}$, vanishes for values of $S$ such that

$$
\frac{J_{1} J_{2}}{S^{2}}=\frac{1}{8} \frac{J_{1}^{2}+J_{2}^{2}+\sqrt{J_{1}^{4}+14 J_{1}^{2} J_{2}^{2}+J_{2}^{4}}}{J_{1} J_{2}} .
$$

The earlier discussion for the additional singularity of $R_{M}$ does not apply in this case, since one can easily show that the points described by (41) do not belong to the black hole region for any values of $J_{1}$ and $J_{2}$. However, we comment in passing that such additional singularities are still related to the vanishing of the determinant of the Hessian of the entropy $S$ with respect to the angular momenta $J_{1}$ and $J_{2}$. Therefore, they still indicate the points where the Hessian vanishes, although they are not situated in the black hole region in this case. We infer from these results that the physical meaning of the divergences of the scalar curvature of the metric $g^{I I}$ for such a 3 -dimensional equilibrium manifold is related to the divergences of the heat capacity at constant angular momenta and to the zeroes of the Hessian of the potential with respect to those momenta, both in the mass and in the entropy representations. On the other side, from the full analysis of the divergences of the generalized response functions, we see that there are other possible points of phase transitions related to divergences of the compressibilities, which appear to be not enclosed by the analysis given with $g^{I I}$. We also comment that we could have used the potential $\Phi=G \equiv M-T S-J_{1} \Omega_{1}-J_{2} \Omega_{2}$ in writing the metric (5) to study the GTD analysis in the $G$ representation, but such investigation would have led to exactly the same results, as it has to be, since the metric (5) is invariant under total Legendre transformations.

To conclude, we observe that in [29] the case of the full Myers-Perry black hole thermodynamics has been investigated using Weinhold and Ruppeiner thermodynamic geometries. The authors proved that both Weinhold and Ruppeiner scalar curvatures only diverge in the extremal limit.

\section{Conclusions}

In this work, we have analyzed the thermodynamics and thermodynamic geometry of different Myers-Perry black holes configurations in five dimensions, classifying them according to the values of the two possible independent angular momenta.

To this end, we followed the approach of Davies for the standard analysis of the thermodynamic properties in different potentials and used the approach of GTD for the thermodynamic geometric investigation. The present work has been carried out with the twofold aim of understanding the phase structure of Myers-Perry black holes in five dimensions and inferring new conclusions on the physical meaning of the metric $g^{I I}$, both in the mass and in the entropy representations.

Our results indicate that the Myers-Perry black holes in five dimensions have a nontrivial phase structure in the sense of Davies. In particular, the analysis of the response functions indicates that both the heat capacities and the compressibilities defined in the $M$ potential diverge at some points, which is usually interpreted as the hallmark of a phase transition. Interestingly, such a behavior is matched by the vanishing of the corresponding Gibbsian response functions in all the cases studied here. Moreover, in the most general case when the two angular momenta vary freely, we have shown that the Gibbsian response functions provide some additional singularities, indicating that the analysis in the $M$ potential is different from that performed in the $G$ potential.

In all the cases studied in this work, the phase transitions are well reproduced by the GTD analysis, while they 
are not reproduced by the thermodynamic geometries of Weinhold and Ruppeiner, whose analysis has been observed to correspond to other approaches (see e.g., [4]). We have also found that the scalar curvature of the metric $g^{I I}$ shows a very similar behavior in the $M$ representation to that of the $S$ representation. In particular, for the cases in which we have only two degrees of freedom we argue that no physical difference has been detected and we have shown that not only the phase transitions indicated by $C_{J}$ are reproduced, but also the ones indicated by divergences of $\kappa_{S}$. Moreover, a detailed analysis of the Gibbsian response functions showed that such divergences correspond to points where $\kappa_{T}$ and $C_{\Omega}$ vanish and change their character. We therefore conclude that for such cases the divergences of the scalar curvature of $g^{I I}$ reproduce the full set of second order phase transitions considered here.

On the other side, it seems that analyzing the general case in which both angular momenta are switched on, that is, a thermodynamic system with three degrees of freedom, some differences might appear. In fact, the phase transitions signaled by $C_{J_{1}, J_{2}}$ are still obtained as curvature singularities in both representations. Nevertheless, the scalar curvature has some additional divergences, which for the case of the $M$ representation can be in the black hole region for appropriate values of the angular momenta and that apparently are not directly related to the response functions of the system. However, we claim that such additional divergences are linked to the vanishing of the Hessian determinant of the potential $M$ with respect to the two angular momenta, and therefore they mark the transition from a stable phase to an unstable one. In our opinion, this means that the physical meaning of the scalar curvature of the metric $g^{I I}$ for thermodynamic systems with three degrees of freedom goes beyond the well-established correspondence with the generalized susceptibilities, that is, second-order derivatives of the potential, encompassing also questions of stability related to their mutual relation, that is, determinants of the Hessian of the potential. This is also supported by the analysis of the scalar curvature in the $S$ representation, which again shows singularities exactly at those points where the Hessian of the entropy with respect to the two angular momenta vanishes, so from the mathematical point of view the situation is basically the same. It is interesting, however, to note that in the $S$ representation such points are not in the black hole region, a direct evidence of the fact that black hole thermodynamics strictly depends on the potential being used. Moreover, in the completely general case, some additional divergences appear when considering the Gibbsian response functions, which are not present in the thermodynamic analysis in the $M$ potential nor are indicated as curvature singularities of $g^{I I}$. The study of such additional singularities goes beyond the scope of this work and may be the matter of further investigation. We also expect to extend this work in the nearest future and find a number of further examples which support (or discard) the interpretation of the thermodynamic metric $g^{I I}$ for thermodynamic systems with 3 degrees of freedom given here.

\section{Acknowledgments}

The authors want to thank Professor H. Quevedo for insightful suggestions. Alessandro Bravetti wants to thank ICRA for financial support.

\section{References}

[1] S. W. Hawking, "Particle creation by black holes," Communications in Mathematical Physics, vol. 43, no. 3, pp. 199-220, 1975.

[2] J. D. Bekenstein, "Black holes and entropy," Physical Review D, vol. 7, pp. 2333-2346, 1973.

[3] J. M. Bardeen, B. Carter, and S. W. Hawking, "The four laws of black hole mechanics," Communications in Mathematical Physics, vol. 31, pp. 161-170, 1973.

[4] G. Arcioni and E. Lozano-Tellechea, "Stability and critical phenomena of black holes and black rings," Physical Review D, vol. 72, no. 10, Article ID 104021, 22 pages, 2005.

[5] P. C. W. Davies, “Thermodynamics of black holes," Reports on Progress in Physics, vol. 41, no. 8, p. 1313, 1978.

[6] C. O. Loustó, "The fourth law of black-hole thermodynamics," Nuclear Physics B, vol. 410, no. 1, pp. 155-172, 1993.

[7] C. O. Lousto, "Erratum," NuclearPhysics B, vol. 449, 433 pages, 1995.

[8] C. O. Lousto, "Some thermodynamic aspects of black holes and singularities," International Journal of Modern Physics D, vol. 6, pp. 575-590, 1997.

[9] R.-G. Cai and J.-H. Cho, "Thermodynamic curvature of the BTZ black hole," Physical Review D, vol. 60, no. 6, Article ID 067502, 4 pages, 1999.

[10] J. Shen, R.-G. Cai, B. Wang, and R.-K. Su, "Thermodynamic geometry and critical behavior of black holes," International Journal of Modern Physics A, vol. 22, no. 1, pp. 11-27, 2007.

[11] R. Banerjee, S. K. Modak, and S. Samanta, "Glassy phase transition and stability in black holes," European Physical Journal C, vol. 70, no. 1, pp. 317-328, 2010.

[12] R. Banerjee, S. K. Modak, and S. Samanta, "Second order phase transition and thermodynamic geometry in Kerr-AdS black holes," Physical Review D, vol. 84, Article ID 064024, 8 pages, 2011.

[13] R. Banerjee and D. Roychowdhury, "Thermodynamics of phase transition in higher dimensional AdS black holes," Journal of High Energy Physics, vol. 2011, article 4, 2011.

[14] R. Banerjee, S. K. Modak, and D. Roychowdhury, "A unified picture of phase transition: from liquid-vapour systems to AdS black holes," Journal of High Energy Physics, vol. 2012, article 125, 2012.

[15] R. Banerjee, S. Ghosh, and D. Roychowdhury, "New type of phase transition in Reissner Nordström-AdS black hole and its thermodynamic geometry," Physics Letters B, vol. 696, pp. 156162, 2011.

[16] R. Banerjee and D. Roychowdhury, "Critical behavior of BornInfeld AdS black holes in higher dimensions," Physical Review $D$, vol. 85, Article ID 104043, 14 pages, 2012.

[17] R. Banerjee and D. Roychowdhury, "Critical phenomena in Born-Infeld AdS black holes," Physical Review D, vol. 85, no. 4, Article ID 044040, 10 pages, 2012.

[18] A. Lala and D. Roychowdhury, "Ehrenfest's scheme and thermodynamic geometry in Born-Infeld AdS black holes," Physical Review D, vol. 86, Article ID 084027, 8 pages, 2012. 
[19] J. W. Gibbs, The Collected Works, vol. 1 of Thermodynamics, Yale University Press, 1948.

[20] C. Carathéodory, "Untersuchungen über die Grundlagen der Thermodynamik," Mathematische Annalen, vol. 67, no. 3, pp. 355-386, 1909.

[21] R. A. Fisher, "Theory of statistical estimation," Mathematical Proceedings of the Cambridge Philosophical Society, vol. 22, no. 5, pp. 700-725, 1925.

[22] C. Radhakrishna Rao, "Information and the accuracy attainable in the estimation of statistical parameters," Bulletin of the Calcutta Mathematical Society, vol. 37, pp. 81-91, 1945.

[23] D. C. Brody and D. W. Hook, "Information geometry in vapourliquid equilibrium," Journal of Physics A, vol. 42, no. 2, Article ID 023001, 33 pages, 2009.

[24] F. Weinhold, "Metric geometry of equilibrium thermodynamics," The Journal of Chemical Physics, vol. 63, no. 6, pp. 24792483, 1975.

[25] G. Ruppeiner, “Thermodynamics: a Riemannian geometric model," Physical Review A, vol. 20, no. 4, pp. 1608-1613, 1979.

[26] G. Ruppeiner, "Riemannian geometry in thermodynamic fluctuation theory," Reviews of Modern Physics, vol. 67, no. 3, pp. 605-659, 1995.

[27] G. Ruppeiner, "Thermodynamic curvature and phase transitions in Kerr-Newman black holes," Physical Review D, vol. 78, no. 2, Article ID 024016, 13 pages, 2008.

[28] J. E. Åman, I. Bengtsson, and N. Pidokrajt, "Flat information geometries in black hole thermodynamics," General Relativity and Gravitation, vol. 38, no. 8, pp. 1305-1315, 2006.

[29] J. E. Åman and N. Pidokrajt, "Geometry of higher-dimensional black hole thermodynamics," Physical Review D, vol. 73, no. 2, Article ID 024017, 7 pages, 2006.

[30] L. Á. Gergely, N. Pidokrajt, and S. Winitzki, "Geometrothermodynamics of tidal charged black holes," European Physical Journal C, vol. 71, no. 3, pp. 1-11, 2011.

[31] H. Quevedo, "Geometrothermodynamics," Journal of Mathematical Physics, vol. 48, no. 1, Article ID 013506, 14 pages, 2007.

[32] H. Quevedo, "Geometrothermodynamics of black holes," General Relativity and Gravitation, vol. 40, no. 5, pp. 971-984, 2008.

[33] H. Quevedo, "Exterior and interior metrics with quadrupole moment," General Relativity and Gravitation, vol. 43, no. 4, pp. 1141-1152, 2011.

[34] H. Quevedo and A. Sanchez, "Geometrothermodynamics of asymptotically Anti-de Sitter black holes," Journal of High Energy Physics, vol. 9, p. 34, 2008.

[35] W. Janke, D. A. Johnston, and R. Kenna, "Geometrothermodynamics of the Kehagias-Sfetsos black hole," Journal of Physics A, vol. 43, no. 42, Article ID 425206, 11 pages, 2010.

[36] H. Quevedo and D. Tapias, "Geometric description of chemicalreactions," submitted, http://arxiv.org/abs/1301.0262.

[37] Y. Han and G. Chen, "Thermodynamics, geometrothermodynamics and critical behavior of $(2+1)$-dimensional black holes," Physics Letters B, vol. 714, no. 2-5, pp. 127-130, 2012.

[38] A. Aviles, A. Bastarrachea-Almodovar, L. Campuzano, and H. Quevedo, "Extending the generalized Chaplygin gas model by using geometrothermodynamics," Physical Review D, vol. 86, Article ID 063508, 10 pages, 2012.

[39] M. E. Rodrigues and Z. A. A. Oporto, "Thermodynamics of phantom black holes in Einstein-Maxwell-dilaton theory," Physical Review D, vol. 85, no. 10, Article ID 104022, 12 pages, 2012.
[40] A. Bravetti and F. Nettel, "Second order phase transitions and thermodynamic geometry: a general approach," submitted, http://arxiv.org/abs/1208.0399.

[41] A. Bravetti, D. Momeni, R. Myrzakulov, and H. Quevedo, "Geometrothermodynamics of higher dimensional black holes," General Relativity and Gravitation, 2013.

[42] J. E. Aman and N. Pidokrajt, "On explicit thermodynamic functions and extremal limits of Myers-Perry black holes," submitted, http://arxiv.org/abs/1004.5550.

[43] R. Emparan and R. C. Myers, "Instability of ultra-spinning black holes," Journal of High Energy Physics, vol. 309, p. 25, 2003.

[44] R. Monteiro, M. J. Perry, and J. E. Santos, "Thermodynamic instability of rotating black holes," Physical Review D, vol. 80, no. 2, Article ID 024041, 18 pages, 2009.

[45] D. Astefanesei, R. B. Mann, M. J. Rodriguez, and C. Stelea, "Quasilocal formalism and thermodynamics of aymptotically flat black objects," Classical and Quantum Gravity, vol. 27, no. 16, Article ID 165004, 22 pages, 2010.

[46] D. Astefanesei, M. J. Rodriguez, and S. Theisen, "Thermodynamic instability of doubly spinning black objects," Journal of High Energy Physics, vol. 1008, article 46, 2010.

[47] V. I. Arnold, Mathematical Methods of Classical Mechanics, Springer, New York, NY, USA, 1989.

[48] H. B. Callen, Thermodynamics and an Introduction to Thermostatics, John Wiley \& Sons, New York, NY, USA, 1985.

[49] A. Bravetti, C. S. Lopez-Monsalvo, F. Nettel, and H. Quevedo, "The conformal metric structure of Geometrothermodynamics," Journal of Mathematical Physics, vol. 54, Article ID 033513, 11 pages, 2013.

[50] R. C. Myers and M. J. Perry, "Black holes in higher-dimensional space-times," Annals of Physics, vol. 172, no. 2, pp. 304-347, 1986.

[51] R. Emparan and H. S. Reall, "Black holes in higher dimensions," Living Reviews in Relativity, vol. 11, p. 6, 2008.

[52] A. Chamblin, R. Emparan, C. V. Johnson, and R. C. Myers, "Charged AdS black holes and catastrophic holography," Physical Review D, vol. 60, no. 6, Article ID 064018, 17 pages, 1999.

[53] A. Chamblin, R. Emparan, C. V. Johnson, and R. C. Myers, "Holography, thermodynamics, and fluctuations of charged AdS black holes," Physical Review D, vol. 60, no. 10, Article ID 104026, 20 pages, 1999. 

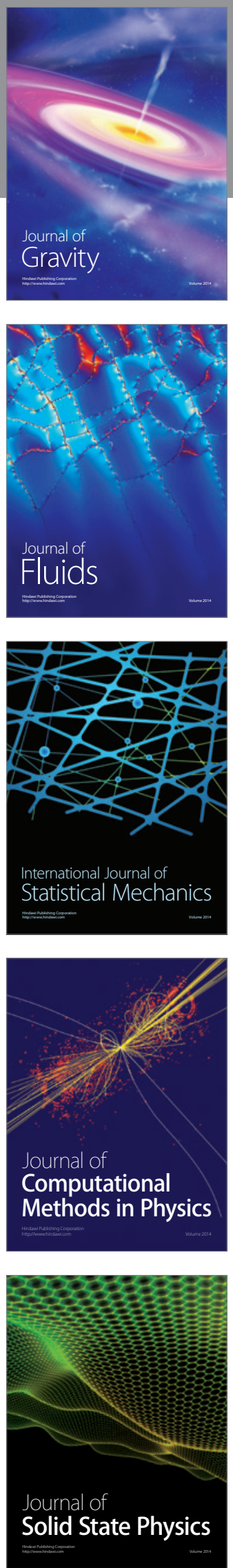

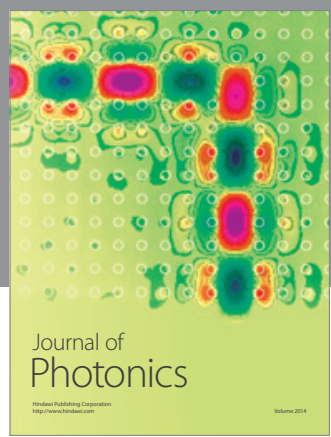

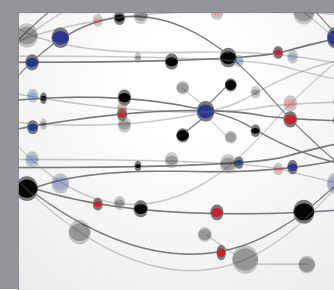

The Scientific World Journal

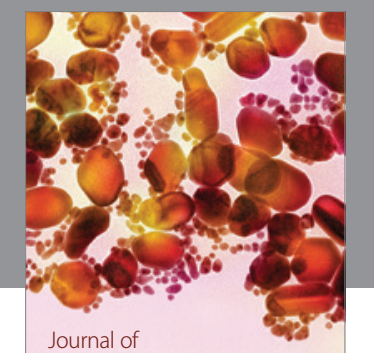

Soft Matter
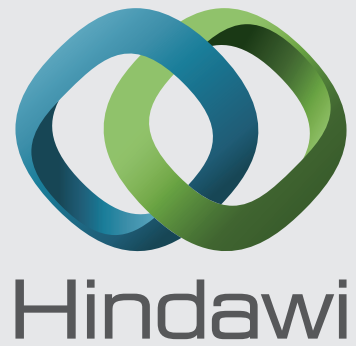

Submit your manuscripts at

http://www.hindawi.com
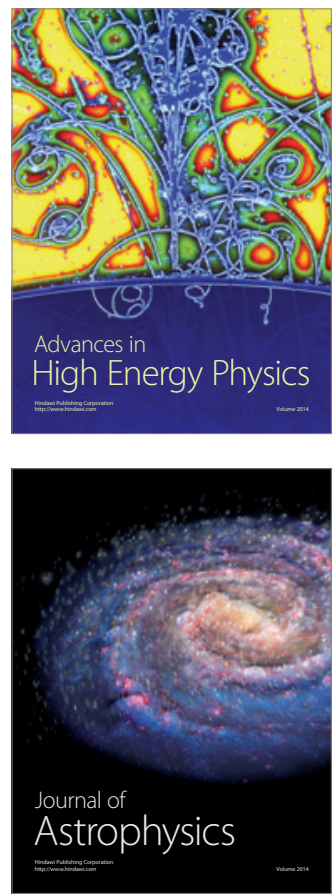
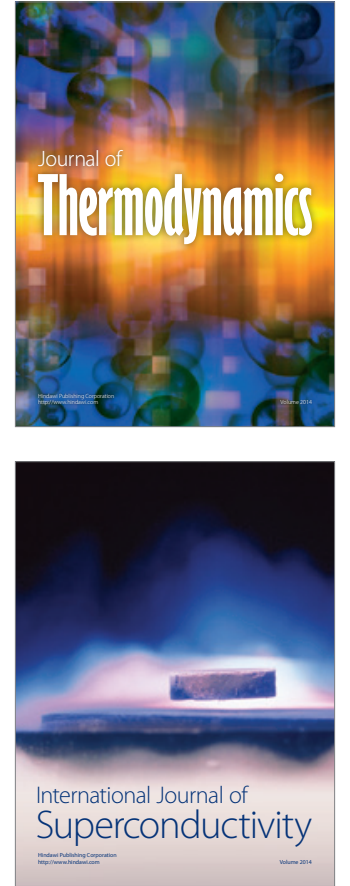
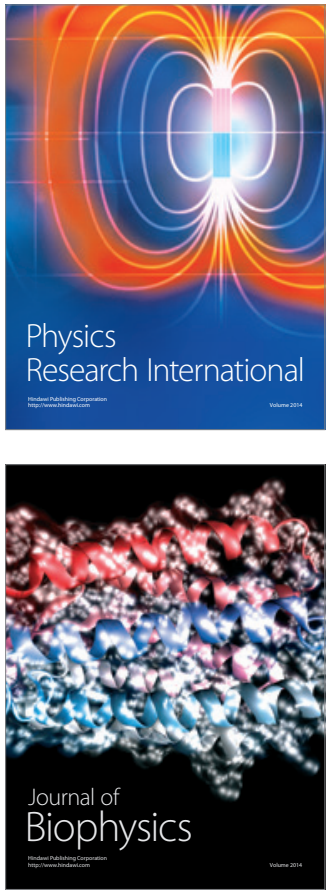
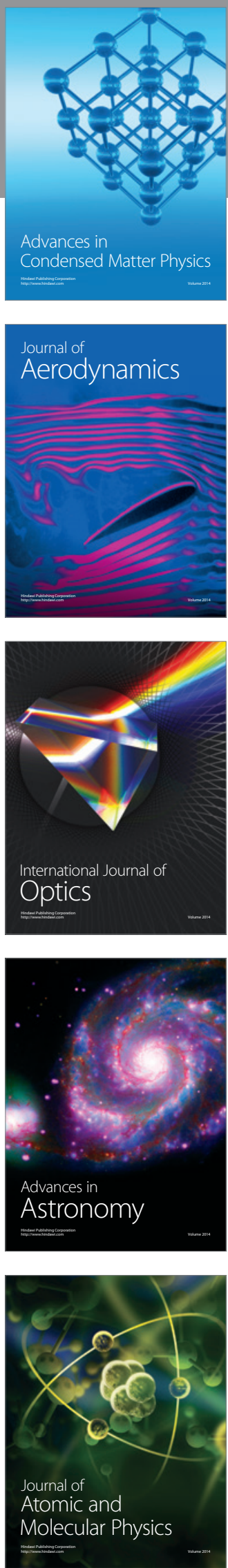